\title{
ASCA OBSERVATIONS OF CATACLYSMIC VARIABLES
}

\author{
MANABU ISHIDA ${ }^{1}$, RYUICHI FUJIMOTO ${ }^{1}$, \\ KEIICHI MA'TSUZAKI ${ }^{2}$ \\ 1. Institute of Space and Astronautical Science \\ 3-1-1 Yoshinodai, Sagamihara, Kanagawa 229, Japan \\ 2. Department of Physics, University of Tokyo \\ 7-3-1 Hongo, Bunkyo-ku, Tokyo 113, Japan
}

\section{Introduction}

ASCA has observed $\sim 20$ cataclysmic variables since launch. In this review, we present the current status of ASCA observations of CVs and scientific results. In Sect. 2, we introduce the scientific instruments of ASCA, and show the list of CVs observed so far. Data analysis and results on magnetic and non-magnetic CVs are described in Sect. 3 and 4, respectively. In Sect. 5, we make a brief summary.

\section{ASCA observations of CVs}

ASCA was launched on 1993 February 20 on a M3S-II rocket from Kagoshima Space Center (Tanaka, Inoue \& Holt 1994). ASCA is the fourth Japanese X-ray astronomy satellite. It is equipped with four equivalent $\mathrm{X}$-ray telescopes, which have large effective area up to $10 \mathrm{keV}$. On the focal plane, two kinds of detector systems, each of which consists of two equivalent detectors, are placed. One is the SIS (Solid-state Imaging Spectrometer) which utilizes a X-ray CCD camera. The other is the GIS (Gas Imaging Spectrometer) which adopts the Gas Scintillation Proportional Counter. Although the effective area of ASCA is significantly smaller than that of Ginga, sensitivity for faint sources is improved by about 100 times because of its imaging capability. The SIS has higher energy resolution and larger effective area in the low energy band. The GIS, on the other hand, has a wider field of view, larger effective area in the high energy band, and higher time resolution. The two detector systems are thus complementary to each other. In Table 1, we show the list of CVs so far observed by ASCA. Up to 1995 June 30 , a total of $28 \mathrm{CVs}$ had been observed. 
TABLE 1. List of targets observed by ASCA to 1995 June

\begin{tabular}{llll|llll}
\hline Type & Target & P.I. & Phase & Type & Target & P.I. & Phase \\
\hline DQ & FO Aqr & Mukai & PV & NM & HT Cas & Mukai & AO-2 \\
NM & SS Cyg & Nousek & PV & NM & V426 Oph & Watson & AO-2 \\
DQ & EX Hya & Ishida & PV & NM & EC1931-5915 & Schlegel & AO-2 \\
AM & EF Eri & Osborne & PV & DQ & TX Col & Wheatley & AO-2 \\
AM & AM Her & Gotthelf & PV & AM & BL Hyi & Fujimoto & AO-2 \\
AM & VV Pup & Ishida & AO-1 & AM & QQ Vul & Mukai & AO-2 \\
NM & VW Hyi & Mauche & AO-1 & AM & RX2107-05 & Schlegel & AO-2 \\
NM & TT Ari & Mauche & AO-1 & NM & U Gem & Szkody & AO-2 \\
AM & V834 Cen & Mukai & AO-1 & DQ & RE0751+14 & Fujimoto & AO-2 \\
AM & BY Cam & Mauche & AO-1 & DQ & GK Per & Mauche & AO-3 \\
DQ & V1223 Sgr & Osborne & AO-1 & AM & AM Her & Ishida & AO-3 \\
DQ & AO Psc & Hellier & AO-2 & NM & Z Cam & Mauche & AO-3 \\
NM & RU Peg & Watson & AO-2 & DQ & TV Col & Mauche & AO-3 \\
NM & GP Com & Marsh & AO-2 & NM & EI UMa & Watson & AO-3 \\
\hline
\end{tabular}

\section{Magnetic cataclysmic variables}

ASCA has observed 17 MCVs (9 AM Her types and 8 DQ Her types) so far. In Fig. 1, we show the phase-average photon-counting SIS spectra of six sources. Owing to the high energy resolution of the SIS, $K \alpha$ emission lines from $\mathrm{Mg}, \mathrm{Si}, \mathrm{S}, \mathrm{Ar}$ and $\mathrm{Fe}$ in hydrogenic and He-like ionization states are resolved. In the AM Her spectrum, the soft blackbody component is detected below $0.6 \mathrm{keV}$.

\subsection{THE CONTINUUM EMISSION}

The hard X-ray continuum emission of MCVs has been known to be very complex. Based on EXOSAT observations, Norton \& Watson (1989) show that the low energy cutoff in the spectra of MCVs cannot be represented by an absorber with a single column density, and they have introduced the partial covering absorber model, which is composed of an absorber with two different hydrogen column densities covering the emission region. This model works well also for the MCV spectra obtained by Ginga in general (Ishida 1991).

However, it became gradually clear that the partial-covering model is sometimes too simplified a parameterization for the low energy cutoff of the MCV spectra. In Fig. 2a is shown the SIS spectrum of PQ Gem $(=\mathrm{RE} 0751+14)$ together with a spectral model. The model consists of a single temperature $(20 \mathrm{keV}$ ) thermal bremsstrahlung (Ishida 1991) covered 

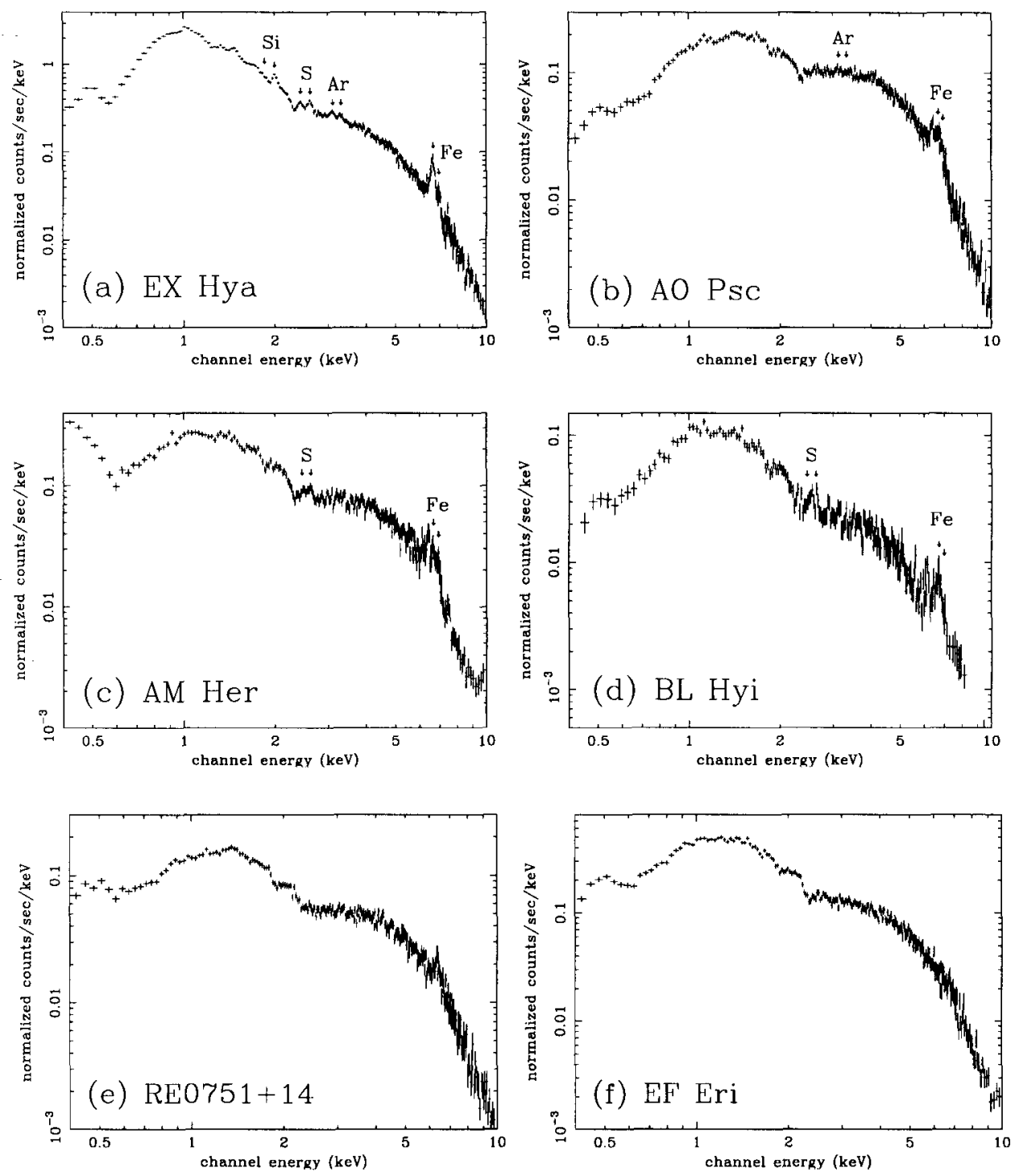

Figure 1. Phase-average spectra of six MCVs taken by the SIS. Detector responses are not deconvolved.

with a series of cold absorbing materials whose hydrogen column densities are $\sim 510^{23} \mathrm{~cm}^{-2}, \sim 310^{22} \mathrm{~cm}^{-2}$ and $<510^{20} \mathrm{~cm}^{-2}$. The covering fractions are $62 \%, 17 \%$ and $21 \%$, respectively. The fit is, however, still not acceptable, with $\chi_{\nu}^{2}=1.40$. This situation is quite similar to FO Aqr (Mukai, 

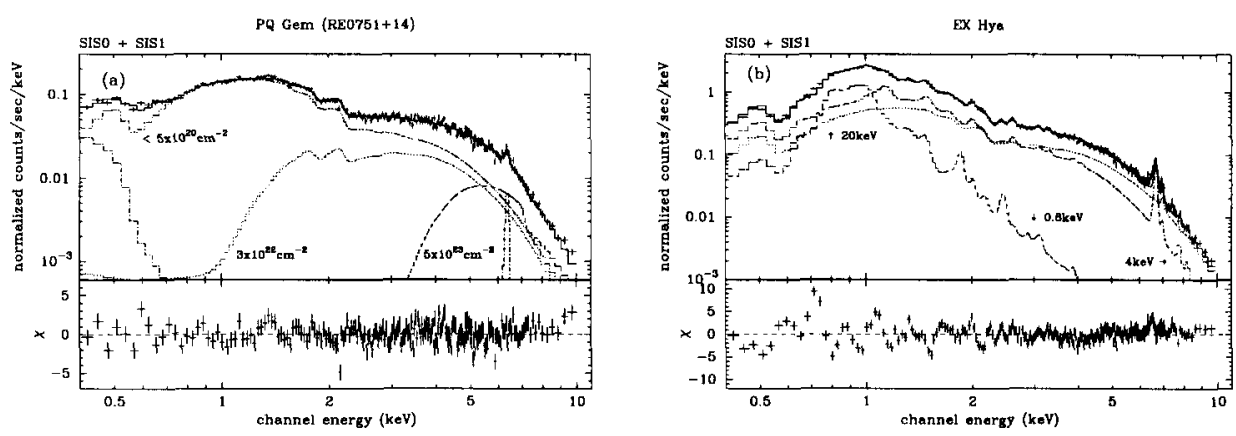

Figure 2. The SIS spectra of (a) PQ Gem and (b) EX Hya. The hydrogen column densities are indicated in (a), and the temperatures of thin thermal plasma are indicated in (b) for each spectral component.

Ishida \& Osborne 1994), and possibly to AM Her (Matsuzaki et al. 1996). Moreover, it becomes clear that the reflection of the hard X-ray emission by the white dwarf surface probably makes a significant contribution to the observed spectrum of AM Her based on the Ginga observation (Beardmore et al. 1995).

In Fig. 2b, we show the spectrum of EX Hya, together with a model. Since the spectrum undergoes very small amount of absorption, $\left(N_{\mathrm{H}}<\right.$ $710^{20} \mathrm{~cm}^{-2}$ ), EX Hya is the best target to investigate the temperature distribution of the post-shock hot plasma. The spectra obtained by the Einstein SSS (Singh \& Swank 1993) and Ginga (Ishida et al. 1994) are both explained well by two optically thin thermal plasma emission components with temperatures of $\sim 1 \mathrm{keV}$ and $\sim 10 \mathrm{keV}$. However, this model does not work for the ASCA SIS spectrum. In Fig. 2b, we have introduced a series of the optically thin thermal plasma emission components with the temperatures of $\sim 0.8 \mathrm{keV}, \sim 4.0 \mathrm{keV}$ and $20 \mathrm{keV}$, but the fit is not acceptable $\left(\chi_{\nu}^{2}=3.04\right)$. This result indicates that we observe a continuous distribution of temperature caused by radiative cooling in the post-shock region.

The hard X-ray continuum of MCV is thus composed of multiple emission components with the photoelectric absorption represented by a continuous distribution of hydrogen column densities and a reflection component. It is therefore very difficult to make a quantitative discussion of the continuum emission. One possible way to resolve this difficulty is to construct an emission model from the post-shock plasma by taking into account the profiles of the temperature and the density (Aizu 1973). This will hopefully enable us to discuss the temperature distribution and the absorption/reflection separately. 


\subsection{DETERMINATION OF THE MASS OF THE WHITE DWARF}

Since the accretion flow becomes highly supersonic close to the white dwarf surface, the temperature just below the shock front is calculated according to the strong shock condition. With the aid of the mass-radius relation, the shock temperature, $T_{\mathrm{S}}$, becomes

$$
k T_{\mathrm{S}}=\frac{3}{8} \frac{G M}{R} \mu m_{\mathrm{H}}=22\left(\frac{M}{0.6 \mathrm{M}_{\odot}}\right)^{4 / 3} \mathrm{keV}
$$

where $\mu$ is the mean molecular weight ( 0.62 for material with solar abundance) and $m_{\mathrm{H}}$ is the mass of a hydrogen atom. So if $T_{\mathrm{S}}$ is obtained, we can determine the mass of the white dwarf. We have developed a method to do this with $K \alpha$ emission lines from heavy elements in the post-shock plasma (Ishida \& Fujimoto 1995; Fujimoto \& Ishida 1995).

The observed line emissions are a superposition of the emission from the entire post-shock plasma. Since the temperature and the density profiles of the post-shock region are solved (e.g. Aizu 1973), the intensity of each emission line is given solely by the boundary conditions. The intensity of the $K \alpha$ line from the element with atomic number $Z$ and the ionization state $z$ is given by

$$
\begin{aligned}
I_{Z, z} & =\frac{1}{4 \pi D^{2}} \int_{0}^{H} A_{Z} \varepsilon_{Z, z}[T(x)] n(x)^{2} S d x \\
& =\frac{A_{Z} S}{4 \pi D^{2}} \int_{T_{\mathrm{B}}}^{T_{\mathrm{S}}} \varepsilon_{Z, z}(T) n(x(T))^{2} \frac{d x}{d T} d T \quad \mathrm{~cm}^{-2} \mathrm{~s}^{-1}
\end{aligned}
$$

here $T_{\mathrm{B}}$ is the temperature at the base of the accretion column, $S$ is the cross section of the accretion column, $D$ the distance, $H$ the height of the shock front measured from the white dwarf surface, $A_{Z}$ is the abundance of the element with atomic number $Z$ relative to the solar value, $\varepsilon_{Z, z}$ is the line emissivity in the case of solar abundance and $n$ is the electron number density. Since $\varepsilon_{Z, z}(T)$ is tabulated in Mewe, Gronenschild \& van den Oord (1985), and the temperature and the density profiles are given by Aizu (1973), the expected intensity is calculated once $T_{\mathrm{S}}, n_{\mathrm{S}}, T_{\mathrm{B}}, D, A_{Z}$ and $S$ are given. Observationally, both hydrogenic and He-like $K \alpha$ lines from the same element are usually detected. Therefore, by taking their intensity ratio, we can eliminate $n_{\mathrm{S}}, D, A_{Z}$ and $S$ from our scheme:

$$
R_{Z}\left(T_{S}, T_{\mathrm{B}}\right)=\frac{I_{Z, Z-1}}{I_{Z, Z-2}}
$$

Now that $R_{Z}$ depends just on the temperatures at the shock front and at the base of the accretion column, we can constrain them if we can measure 
TABLE 2. $T_{S}$ and mass of the white dwarf.

\begin{tabular}{lccl}
\hline Star & $T_{\mathrm{S}}(\mathrm{keV})$ & Mass $\left(\mathrm{M}_{\odot}\right)$ & Elements Used \\
\hline EX Hya & $15 \pm 2$ & $0.47 \pm 0.04$ & $\mathrm{Si}, \mathrm{S}, \mathrm{Ar}, \mathrm{Fe}$ \\
AO Psc & $12 \pm 4$ & $0.4 \pm 0.1$ & $\mathrm{Ar}, \mathrm{Fe}$ \\
\hline
\end{tabular}

TABLE 3. Detected emission lines

\begin{tabular}{lccccc}
\hline & $\mathrm{Mg}$ & $\mathrm{Si}$ & $\mathrm{S}$ & $\mathrm{Ar}$ & $\mathrm{Fe}$ \\
\hline EX Hya & $*$ & $*$ & $*$ & $*$ & $*$ \\
V1223 Sgr & $*$ & $*$ & $*$ & $*$ & $*$ \\
AM Her & - & $*$ & $*$ & - & $*$ \\
BL Hyi & - & $*$ & $*$ & - & $*$ \\
AO Psc & - & - & - & $*$ & $*$ \\
FO Aqr & - & - & - & - & $*$ \\
EF Eri & - & - & - & - & $*$ \\
PQ Gem & - & - & - & - & - \\
\hline$* \cdots$ detection $(>90 \%)$ & & &
\end{tabular}

the line intensity ratio of at least two elements. This method is currently successful for EX Hya and AO Psc, for which the results are summarized in Table 2.

Note that if the magnetic field of the white dwarf is as strong as $10 \mathrm{MG}$, cyclotron emission makes a significant contribution to the cooling of the post-shock plasma ( $\mathrm{Wu}$, Chanmugam \& Shaviv 1994). To take this effect correctly into account, we have to know the absolute value of the density at the shock, for which we have to await ASTRO-E. Our method is thus applicable now only for intermediate polars. We are going to apply our method to other intermediate polars. Note also that the $K \alpha$ emission lines are not detected from all the MCVs. The reason for this is discussed in the next section.

\subsection{PHOTOIONIZATION EFFECT}

As we have shown in Fig. 1, $K \alpha$ emission lines from heavy elements are detected from several sources. In Table 3, we show a list of detected emission lines. From this table, it is understood that the emission lines from light elements are more difficult to be detected. One possible explanation is that the plasma is still too hot for completely ionized light elements to start 
to recombine with electrons, even at the base of the accretion column. Another possiblity is to assume abundance anomalies; heavier elements are more abundant than lighter elements in general, which is rather ad hoc.

Both these ideas are, however, faced with difficulty in explaining the spectrum of $\mathrm{PQ}$ Gem (Fig. 1e). In the PQ Gem spectrum, only $K \alpha$ emission line is detected at $6.4 \mathrm{keV}$ with an equivalent width (EW) of $\sim 100 \mathrm{eV}$, which originates from the pre-shock cold accretion column and the surface of the white dwarf via fluorescence. The temperature of the post-shock plasma in $\mathrm{PQ}$ Gem is measured to be $20 \mathrm{keV}$ by the Ginga observation (Ishida 1991). At this temperature, the $K \alpha$ emission line from hydrogenic iron should be detected with an $\mathrm{EW}$ of $200 \mathrm{eV}$. So the plasma is cool enough to radiate the emission line, but the line is absent due to other reasons. The abundance anomaly is also rejected, because the EW of the fluorescent iron $K \alpha$ line indicates that the abundance of the accreting matter is close to the solar value (Beardmore et al. 1995).

The last possibility is the photoionization effect in the post-shock hot plasma. In the low density plasma $\left(n_{\mathrm{e}}<10^{10} \mathrm{~cm}^{-3}\right)$, the ionization equilibrium of iron is determined by a balance between electron collisional ionization and radiative recombination, and photoionization can be neglected. The condition for this is that

$$
S_{Z, z} n_{\mathrm{e}} \gg \int_{E_{0}}^{\infty} f(E) \sigma_{Z, z}(E) d E
$$

where $S_{Z, z}$ is the electron-collisional ionization rate coefficient (in $\mathrm{cm}^{3} \mathrm{~s}^{-1}$; Mewe \& Gronenschild 1981 and references therein), $E_{0}$ is the edge energy, $f$ is the photon flux, and $\sigma_{Z, z}(E)$ is the photoionization cross section (Band et al. 1990). It is understood from equation (4) that the photoinization becomes dominant for high density, because $f \propto n^{2}$.

In Fig. 3, we show a distribution map of the collisional ionizationdominant and photoionization-dominant regions on the density versus temperature plane for hydrogenic and He-like iron. Here we consider a cylinder with height and diameter of $1 \%$ of the white dwarf radius. Evaluation is made at the center of this cylinder filled with uniform plasma with the density and the temperature indicated in the abscissa and the ordinate, respectively. The track of the density and the temperature indicated by the Aizu model in the case of $k T_{\mathrm{S}}=20 \mathrm{keV}$ and $n_{\mathrm{S}}=10^{16} \mathrm{~cm}^{-3}$ is shown. For hydrogenic and He-like iron the plasma, first in the collision-dominant region, gradually moves into the photoinization-dominant region as it descends the accretion column.

If photoionization becomes important, the ionization distribution shifts to higher ionization degree than what is expected from the collisional ionization only, and the He-like and hydrogenic elements will eventually be 


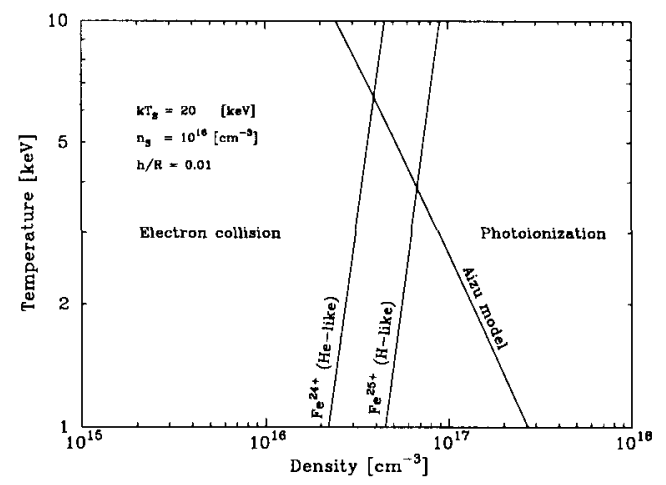

Figure 3. The collisional ionization-dominant and the photoionization-dominant region in the density and the temperature plane. Note that the result is dependent on the geometry (see text).
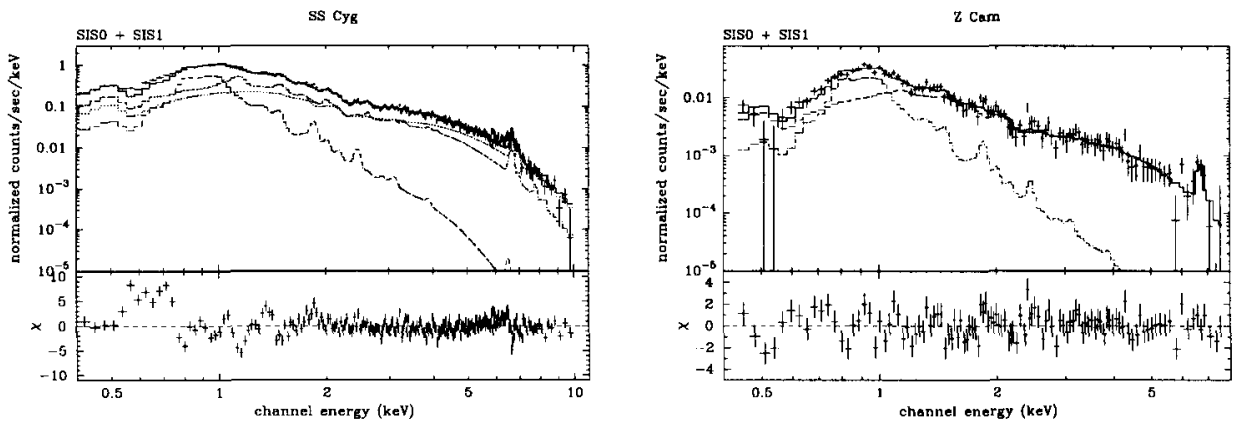

Figure 4. The SIS spectra of SS Cyg and Z Cam

completely ionized, leading to the absence of the emission lines. Note that this effect is more significant for light elements, because, although both $S_{Z, z}$ and $\sigma_{Z, z}$ are both proportional to $Z^{-2}$, the photon flux is always greater at lower energies. Also, $K \alpha$ lines of lighter elements are emitted from lower portions of the accretion column, where the density is higher. Depression of emission lines from light elements can be attributed to the photoionization effect in the post-shock plasma.

\section{Non-magnetic cataclysmic variables}

\subsection{SS CYG}

In Fig. 4, we show the SIS spectrum of SS Cyg. At the time of the ASCA observation (1993 May 27), SS Cyg was nearly at the end of the peak of a so-called anomalous outburst (Nousek et al. 1994). It is well known from EXOSAT observations (Watson, King \& Heise 1985) that the hard X-ray flux drops when the source enters into outburst. In the ASCA observation, 
the flux is measured to be $2.710^{-11} \mathrm{erg} \mathrm{s}^{-1} \mathrm{~cm}^{-2}$ in the $2 \ldots 10 \mathrm{keV}$ range, which is smaller than that measured by Ginga $\left(8.310^{-11} \mathrm{erg} \mathrm{s}^{-1} \mathrm{~cm}^{-2}\right.$ in quiescence) by a factor of 3 (Yoshida, Inoue \& Osaki 1992).

Yoshida et al. (1992) have introduced a two temperature $(\sim 7 \mathrm{keV}$ and $\sim 45 \mathrm{keV}$ ) plasma emission model based on Ginga observations. Because of the high energy resolution of the SIS, the spectrum can no longer be fit with this model. In Fig. 4, we have introduced a three temperature $(\sim 0.8 \mathrm{keV}$, $\sim 3 \mathrm{keV}$ and $\sim 20 \mathrm{keV}$ ) plasma model, but there still remain systematic residuals below $2 \mathrm{keV}$, which correspond to the $K \alpha$ emission lines from $\mathrm{Ne}, \mathrm{Mg}$ and Si. The situation is quite similar to EX Hya (Sect. 3.1). It is remarkable that the EW of the iron $K \alpha$ emission line is $735 \pm 90 \mathrm{eV}$, which is twice as large as that in quiescence $(355 \pm 70 \mathrm{eV})$. This means the average temperature of the plasma in outburst is significantly lower than that in quiescence, because the $\mathrm{EW}$ of the iron $K \alpha$ emission line is a monotonically decreasing function of the plasma temperature above $1 \mathrm{keV}$. Assuming solar abundance, the average plasma temperature is $10 \mathrm{keV}$ and $18 \mathrm{keV}$ during the ASCA and the Ginga observation, respectively. The hard X-ray flux is proportional to $\sqrt{T} n_{\mathrm{e}}^{2} V$, where $V$ is the volume of the optically thin boundary layer. Considering drops of the temperature and the flux by a factor of 2 and 3 , respectively, during the outburst, the emission measure $n_{\mathrm{e}}^{2} V$ decreases by a factor of 2 . However, the density is considered to be higher than in the quiescence. This means that the volume of the boundary layer should significantly shrink when the source enters into outburst.

\subsection{Z CAM}

Z Cam was observed in 1995 March $8 \ldots 12$, also nearly at the end of the peak of the outburst. The SIS spectrum (Fig. 4 right) is complex like that of SS Cyg, and we have introduced a two temperature $(\sim 0.8 \mathrm{keV}$ and $\sim 7 \mathrm{keV})$ plasma emission model. The fit is, however, not acceptable $\left(\chi_{\nu}^{2}=1.34\right)$. The observed flux is $6.510^{-13} \mathrm{erg} \mathrm{s}^{-1} \mathrm{~cm}^{-2}$ in the $2 \ldots 10 \mathrm{keV}$ energy band.

\section{Summary}

With the emission line information obtained by ASCA, we have discussed the mass of the white dwarf and the possibility of the photoionization effect for MCVs.

ASCA observations have revealed that the hard X-rays from the boundary layer in dwarf novae are the emission from a multi-temperature plasma. The ASCA observations can potentially improve the understanding of the structure of the boundary layer. 


\section{References}

1. Aizu, K., 1973, Prog. Theoret. Phys., 49, 1184

2. Band, I.M., Trzhaskovskaya, M.B., Verner, D.A., Yakovlev, D.G., 1990, A\&A, 237, 267

3. Beardmore, A.P., Done, C., Osborne, J.P., Ishida, M., 1995, MNRAS, 272, 749

4. Fujimoto, R., Ishida, M., 1995, in "Magnetic Cataclysmic Variables", eds D. Buckley, B. Warner, ASP Conf. Ser. 85, p136

5. Ishida, M., 1991, Ph.D. thesis, University of Tokyo/ ISAS RN 505

6. Ishida, M., Makishima, K., Mukai, K., Masai, K., 1994, MNRAS, 266, 367

7. Ishida, M., Fujimoto, R., 1995, in "Magnetic Cataclysmic Variables", eds D. Buckley, B. Warner, ASP Conf. Ser. 85, p132

8. Matsuzaki, K., Ishida, M., Fujimoto, R., Kitamura, H., 1996, in "Proceedings of The 11th Colloquium on UV and X-ray Spectroscopy of Astrophysical and Laboratory Plasma", Universal Academy Press, Tokyo, in press

9. Mewe, R., Gronenschild, E.H.B.M., 1981, A\&A Suppl., 45, 11

10. Mewe, R, Gronenschild, E.H.B.M., van den Oord, G.H.J., 1985, A\&A Suppl., 62, 197

11. Mukai, K., Ishida, M., Osborne, J.P., 1994, PASJ, 46, L87

12. Norton, A.J., Watson, M.G., 1989, MNRAS, 237, 853

13. Nousek, J.A., Baluta, C.J., Corbet, R.H.D., et al., 1994, ApJ, 436, L19

14. Singh, J., Swank, J., 1993, MNRAS, 262, 1000

15. Tanaka, Y., Inoue, H., Holt, S.S., 1994, PASJ, 46, L37

16. Watson, M.G., King, A.R., Heise, J., 1985, Space Sci. Rev., 40, 127

17. Wu, K.W., Chanmugam, G., Shaviv, G., 1994, ApJ, 426, 664

18. Yoshida, K., Inoue, H., Osaki, Y., 1992, PASJ, 44, 537 